Case Report

\title{
Giant Popliteal Artery Aneurysm: Case Report and Review of the Literature
}

\author{
Christos Verikokos, ${ }^{1}$ Georgios Karaolanis, ${ }^{1}$ Mikes Doulaptsis, ${ }^{1}$ Georgios Kouvelos, ${ }^{2}$ \\ Aikaterini Kotzadimitriou, ${ }^{1}$ Viktoria-Varvara Palla, ${ }^{3}$ and Christos Klonaris ${ }^{1,2}$ \\ ${ }^{1}$ 2nd Department of Surgery, Vascular Surgery Unit, Laiko General Hospital, Medical School of Athens, \\ Agiou Thoma 17, 11527 Athens, Greece \\ ${ }^{2} 1$ st Department of Surgery, Vascular Surgery Unit, Laiko General Hospital, Medical School of Athens, Athens, Greece \\ ${ }^{3}$ Department of Gynecology, G. Genimatas General Hospital, Medical School of Athens, Athens, Greece
}

Correspondence should be addressed to Georgios Karaolanis; drgikaraolanis@gmail.com

Received 5 May 2014; Accepted 1 August 2014; Published 21 August 2014

Academic Editor: Atila Iyisoy

Copyright (C) 2014 Christos Verikokos et al. This is an open access article distributed under the Creative Commons Attribution License, which permits unrestricted use, distribution, and reproduction in any medium, provided the original work is properly cited.

Popliteal artery aneurysms (PAAs) are rare in general population but represent the second most common peripheral arterial aneurysms following those located in the aortoiliac segment. They usually affect men over 60 years old with established cardiovascular disease caused by atherosclerosis. Other more unusual conditions such as trauma, congenital popliteal aneurysm, mycotic aneurysm, inflammatory arteritis, or popliteal entrapment are responsible. The authors report the first ever case of a male diagnosed with chronic renal failure with giant popliteal artery aneurysm. We have successfully resected the aneurysm and revascularized with synthetic graft.

\section{Introduction}

Popliteal artery aneurysms (PAAs) represent the second most common peripheral arterial aneurysms following those located in the aortoiliac segment. They account for approximately $70 \%$ of all peripheral aneurysms with an incidence estimated to be less than $0.1 \%$ [1]. They are more common in men over 60 years old with established cardiovascular disease and they are often associated with contralateral PAAs and abdominal aortic aneurysms [2]. PAAs are typically caused by atherosclerosis but they have also been observed in other more unusual conditions such as trauma, congenital popliteal aneurysm, mycotic aneurysm, inflammatory arteritis, or popliteal entrapment [3]. Huge PAAs, because of their anatomical position, size, and common complications, pose a therapeutic challenge for the vascular surgeon. We present a case of a male with a giant PAA treated surgically.

\section{Case Report}

A 63-year-old male was admitted to our department complaining of pain and swelling behind his left knee aggravating throughout the last month. The patient had a history of chronic renal failure under hemodialysis, hypertension, and diabetes mellitus and was a heavy smoker. He had undergone saphenous vein ablation on the right leg due to significant venous insufficiency. There was no history of any trauma or local/systemic infection.

On examination, a palpable pulsatile mass in the upper popliteal fossa was revealed. Left distal pulses were weak, while the ankle brachial index (ABI) for the left and right limb was 0.8 and $>1$, respectively.

Magnetic resonance imaging (MRI) revealed the presence of a popliteal mass with a cephalocaudal diameter of $11 \mathrm{~cm}$ (Figure 1). Color duplex ultrasonography demonstrated a huge PAA with an uneven echogenic collection inside and 


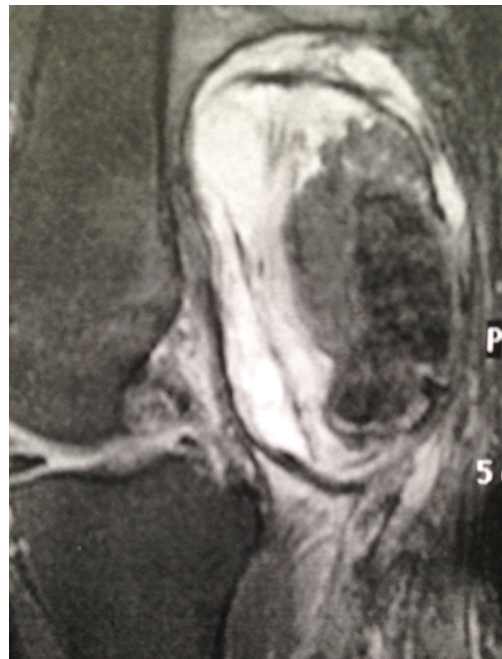

(a)

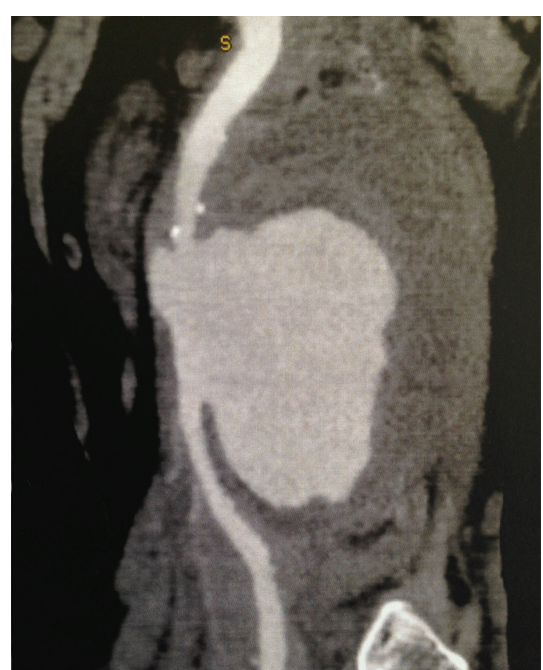

(b)

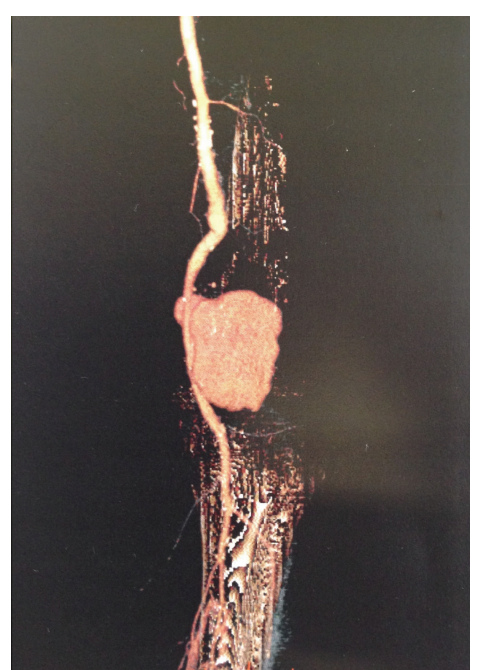

(c)

FIGURE 1: (a) Magnetic resonance imaging (MRI) showing the huge popliteal artery aneurysm. (b) Computed tomography angiography (CTA) depicting the huge aneurysm with significant thrombus inside the aneurysm sac. (c) 3D reconstruction of CTA showing the aneurysm with the inflow and outflow vessels.

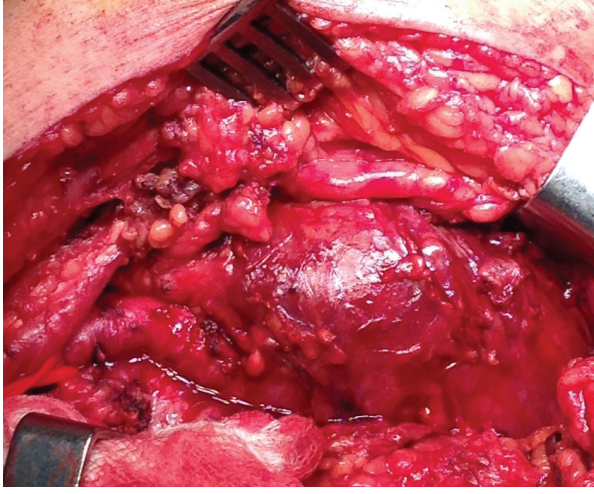

(a)

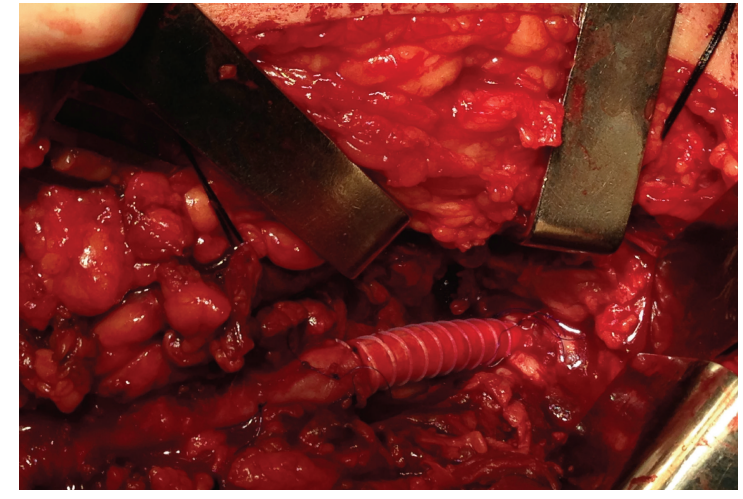

(b)

FIGURE 2: (a) Giant popliteal artery aneurysm intraoperatively. (b) Revascularization with polytetrafluoroethylene (PTFE) graft.

a thrombosed left popliteal vein, along with diminished flow of the tibial arteries. Computed tomography angiogram (CTA) depicted a multilobulated mass $(5.6 \times 5.6 \times 14.3 \mathrm{~cm})$ extending from the midthigh to popliteal fossa, with the presence of thrombus inside (Figure 1).

"After discussing with the patient his therapeutic options for an open or an endovascular procedure, he decided for an open approach, as a more durable solution, despite the greater perioperative risk."

\section{Procedure}

With medial approach, the distal superficial femoral artery and the distal popliteal artery were exposed to allow proximal and distal arterial control (Figure 2). The aneurysm sac was then opened and both the thrombus and the wall were removed and sent for histological examination. A bypass grafting was performed from the distal femoral artery to the distal popliteal artery above the knee with the use of a polytetrafluoroethylene (PTFE) graft in an end-to-side fashion (Figure 2). The patient had an uneventful postoperative course and was discharged in good general condition with pulsatile pedal pulses. At the 6-month follow-up, the patient was well and suffered no complications. Duplex scan showed excellent graft patency with no other complications.

\section{Discussion}

PAA is the most common peripheral aneurysm presenting bilaterally in $50 \%$ and coexisting with abdominal aortic aneurysm (AAA) in $50 \%$ of cases. Although $80 \%$ are asymptomatic at the time of diagnosis [4], they become 
symptomatic at a rate of $14 \%$ per year [5]. In the literature, five cases of giant PAA treated have been reported: one case of bilateral popliteal artery entrapment syndrome [6], one of a posttraumatic popliteal artery pseudoaneurysm, occurring during a soccer game [7], and another one in a young child of congenital aetiology [8]. There is also another case of a ruptured giant popliteal aneurysm occurring in an 84-yearold male who presented with a multilobulated mass on the left leg and a large mass on the right leg. In this case, the authors proceeded to the evacuation of the multilobulated mass of the left leg without surgical revascularization because of the absence of symptoms and the presence of an adequate collateral blood flow. On the other limb, ligation and bypass with nonreversed greater saphenous vein were performed [9]. Additionally, a case of an 88-year-old woman with a PAA of $11 \mathrm{~cm}$ has been reported. The patient was unfit for open surgery because of her advanced age and the concomitant comorbidities, so an endovascular approach was elected. The results were satisfactory and the woman has an uneventful postoperative course [10].

Large aneurysms are at risk of serious complications such as arterial embolization, thrombosis, and, less commonly, rupture. Although the indications for repair of PAAs are not well defined, a diameter greater than $2 \mathrm{~cm}$ to $3 \mathrm{~cm}$, especially for aneurysms with a significant thrombus load or with chronic distal tibial artery embolic occlusion, is an acceptable indication for intervention [11, 12]. Patients with severe claudication, resting pain, and tissue loss are considered for elective repair, as are patients who have symptoms of local compression. Indications for asymptomatic patients and for those with mild-to-moderate claudication are less well defined. Recently, the approach to repair these aneurysms has become a subject of debate, particularly with the increased utilization of endovascular techniques [13]. Different studies comparing both techniques have been published. Tsilimparis et al. in a retrospective analysis presented the data of studies published in the past 25 years and came to the conclusion that open surgical repair remains the gold standard but the endovascular repair is being performed more commonly with acceptable results [14]. Furthermore, Galiñanes et al. in a retrospective analysis of 2962 patients showed that the rates of reinterventions during 30 and 90 days after initial procedure were considerably greater after endovascular procedure than after open surgery [15]. In addition, open approach appeared to convey greater durability and greater cost benefit than those of endovascular approach $[15,16]$. An important point was that this approach was suggested for patients of a good health status. They may benefit from the durability of an open repair and avoid the higher reintervention rates associated with an endovascular approach [15]. Pulli et al. analysed early and follow-up results of the treatment of PAA performed with open surgical repair or with endovascular exclusion with endografts, involving seven Italian vascular centers. Similarly, they reported the same results for the two procedures [11]. In our case, based on patient's age and the compromised distal run-off, we decided to proceed with an open surgical approach to achieve a more durable result and avoid the risk for reintervention. Moreover, due to the huge size of the aneurysm followed by mechanical compression of adjacent structures, the surgical exploration with hematoma evacuation and arterial repair was the most appropriate approach. Nevertheless, in young patients, limitation of endovascular technique consists of the inability to obtain cultures or biopsies in order to diagnose infection or congenital abnormality.

In conclusion, giant PAA is a rare entity with very limited number of cases published so far. Compression issues and patient's characteristics should be meticulously considered in treatment decision. Open surgical approach seems to comprise a durable and adequate therapeutic strategy in such cases.

\section{Consent}

A written informed consent was obtained from the patient for publication of this case report and accompanying images. A copy of the written consent is available for review.

\section{Conflict of Interests}

The authors declare that there is no conflict of interests regarding the publication of this paper.

\section{Authors' Contribution}

Christos Verikokos and Georgios Karaolanis equally contributed.

\section{References}

[1] M. Antonello, P. Frigatti, P. Battocchio et al., "Endovascular treatment of asymptomatic popliteal aneurysms: 8-year concurrent comparison with open repair," Journal of Cardiovascular Surgery, vol. 48, no. 3, pp. 267-274, 2007.

[2] B. Aulivola, A. D. Hamdan, C. N. Hile et al., "Popliteal artery aneurysms: a comparison of outcomes in elective versus emergent repair," Journal of Vascular Surgery, vol. 39, no. 6, pp. 1171-1177, 2004.

[3] T. R. Vogel, V. Y. Dombrovskiy, J. L. Carson, P. B. Haser, S. F. Lowry, and A. M. Graham, "Infectious complications after elective vascular surgical procedures," Journal of Vascular Surgery, vol. 51, no. 1, pp. 122-129, 2010.

[4] R. B. Galland, "History of the management of popliteal artery aneurysms," European Journal of Vascular and Endovascular Surgery, vol. 35, no. 4, pp. 466-472, 2008.

[5] J. E. Cross and R. B. Galland, "Part one: for the motion. Asymptomatic popliteal artery aneurysms (less than $3 \mathrm{~cm}$ ) should be treated conservatively," European Journal of Vascular and Endovascular Surgery, vol. 41, no. 4, pp. 445-448, 2011.

[6] C. B. Park, D. G. Yoo, and C. W. Kim, "Bilateral popliteal artery entrapment syndrome," The Korean Journal of Thoracic and Cardiovascular Surgery, vol. 40, pp. 136-139, 2007.

[7] S. Y. Lee, S. Lee, and C. Lee, "Traumatic popliteal artery pseudoaneurysm developed during a soccer game," Korean Journal of Thoracic and Cardiovascular Surgery, vol. 44, no. 4, pp. 298-300, 2011.

[8] X. Gao, L. Qi, B. Chen, H. Yu, J. Li, and J. Zhang, "A rare case of giant popliteal artery aneurysm in a young adult," Vascular, vol. 19 , no. 6 , pp. 342-345, 2011. 
[9] J. A. Tschen, K. Samakar, S. T. Patel, and A. M. Jr. AbouZamzam, "Asymptomatic ruptured giant popliteal aneurysm," Journal of Vascular Surgery, vol. 58, no. 4, article 1090, 2013.

[10] A. Ponton, I. Garcia, E. Arnaiz et al., "Endovascular repair of a ruptured giant popliteal artery aneurysm," Annals of Vascular Surgery, vol. 23, no. 3, article 412, 2009.

[11] R. Pulli, W. Dorigo, P. Castelli et al., "A multicentric experience with open surgical repair and endovascular exclusion of popliteal artery aneurysms," European Journal of Vascular and Endovascular Surgery, vol. 45, no. 4, pp. 357-363, 2013.

[12] A. Zimmermann, T. Schoenberger, J. Saeckl et al., "Eligibility for endovascular technique and results of the surgical approach to popliteal artery aneurysms at a single center," Annals of Vascular Surgery, vol. 24, no. 3, pp. 342-348, 2010.

[13] G. Guzzardi, R. Fossaceca, P. Cerini et al., "Endovascular treatment of popliteal artery aneurysms: preliminary results," Radiologia Medica, vol. 118, no. 2, pp. 229-238, 2013.

[14] N. Tsilimparis, A. Dayama, and J. J. Ricotta II, "Open and endovascular repair of popliteal artery aneurysms: tabular review of the literature," Annals of Vascular Surgery, vol. 27, no. 2, pp. 259-265, 2013.

[15] E. L. Galiñanes, V. Y. Dombrovskiy, A. M. Graham, and T. R. Vogel, "Endovascular versus open repair of popliteal artery aneurysms: Outcomes in the US medicare population," Vascular and Endovascular Surgery, vol. 47, no. 4, pp. 267-273, 2013.

[16] T. Sachs, F. Pomposelli, A. Hamdan, M. Wyers, and M. Schermerhorn, "Trends in the national outcomes and costs for claudication and limb threatening ischemia: angioplasty vs bypass graft," Journal of Vascular Surgery, vol. 54, no. 4, pp. 10211031, 2011. 


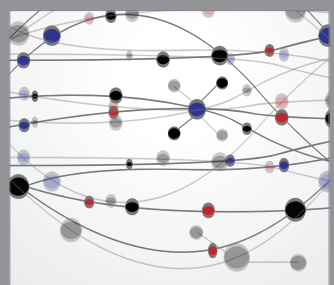

The Scientific World Journal
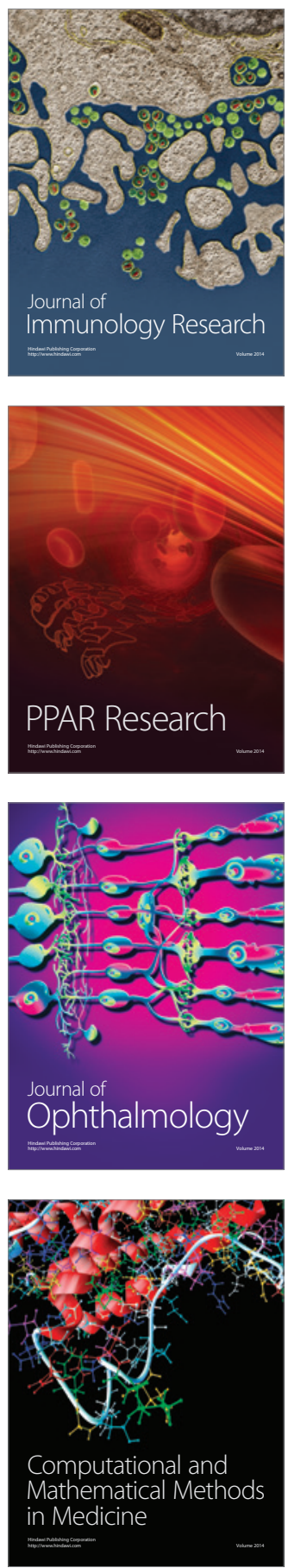

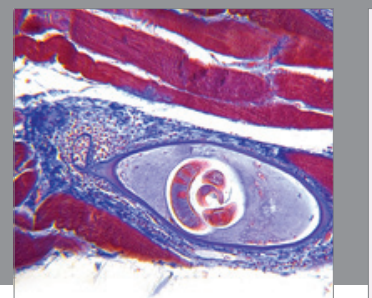

Gastroenterology

Research and Practice
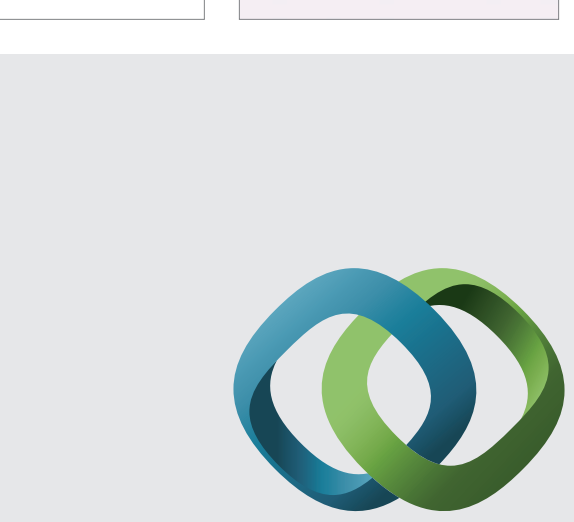

\section{Hindawi}

Submit your manuscripts at

http://www.hindawi.com
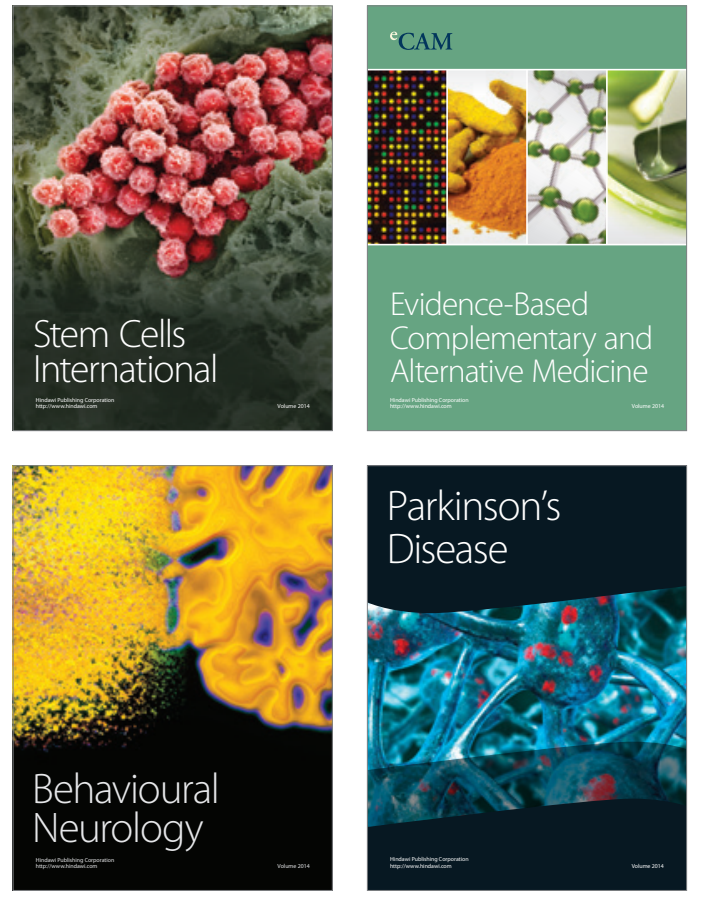
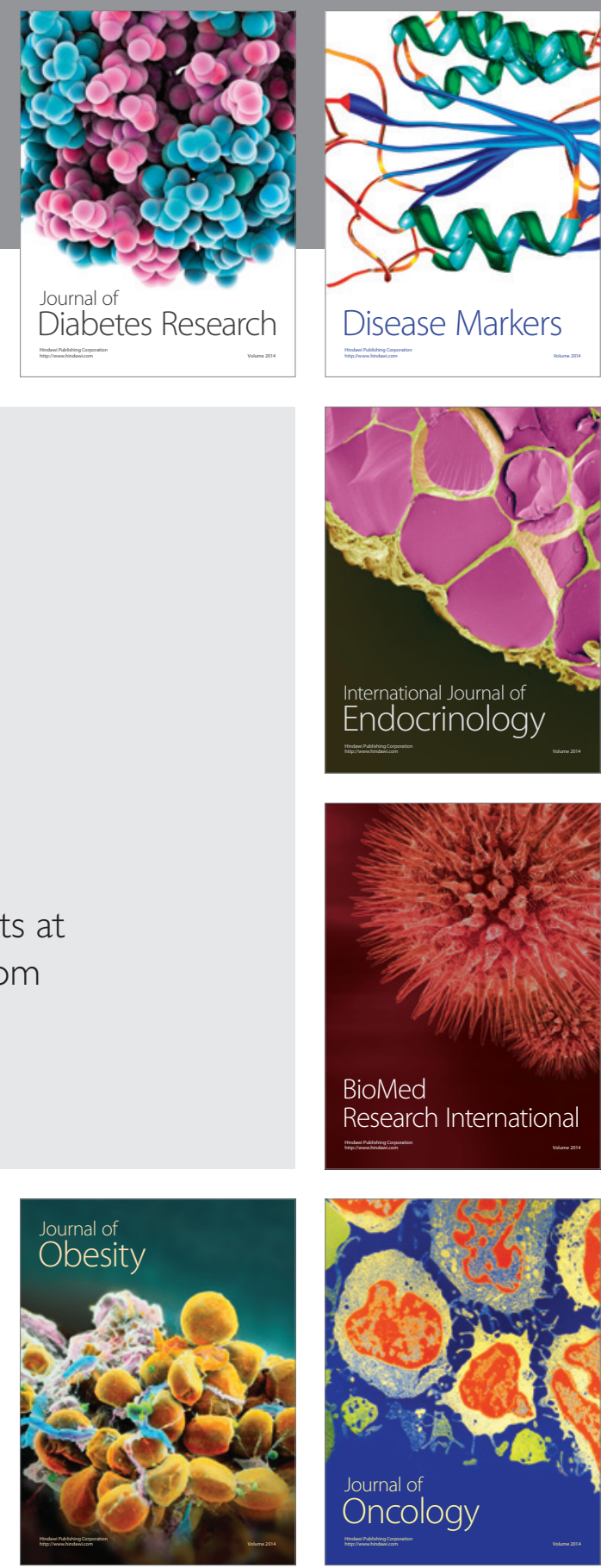

Disease Markers
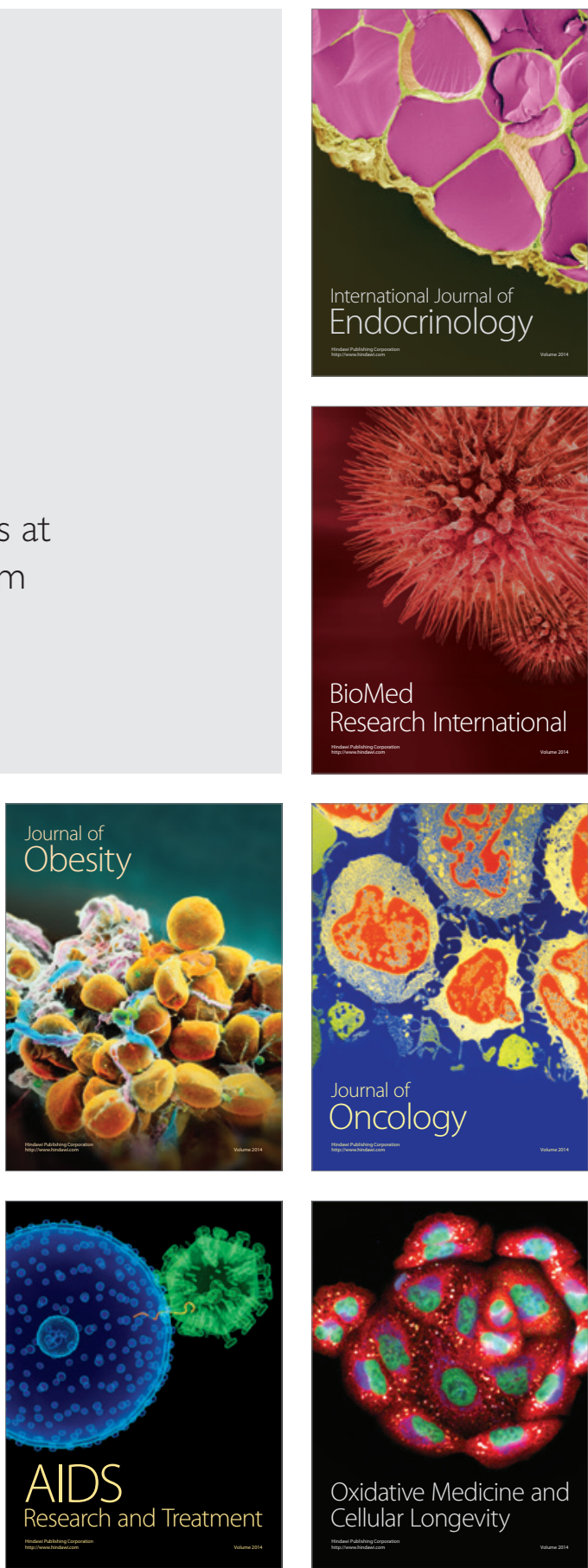